\title{
A Comparison of National Water Model Retrospective Analysis Snow Outputs at SNOTEL Sites Across the Western U.S.
}

\author{
Irene Garousi-Nejad ${ }^{1}$ and David Tarboton ${ }^{1}$ \\ ${ }^{1}$ Utah State University
}

December 13, 2021

\begin{abstract}
This study compares the U.S. National Water Model (NWM) reanalysis snow outputs to observed snow water equivalent (SWE) and snow-covered area fraction (SCAF) at SNOTEL sites across the Western U.S. SWE was obtained from SNOTEL sites, while SCAF was obtained from MODIS observations at a nominal $500 \mathrm{~m}$ grid scale. Retrospective NWM results were at a 1000 $\mathrm{m}$ grid scale. We compared results for SNOTEL sites to gridded NWM and MODIS outputs for the grid cells encompassing each SNOTEL site. Differences between modeled and observed SWE were attributed to both model errors, as well as errors in inputs, notably precipitation and temperature. The NWM generally under-predicted SWE, partly due to precipitation input differences. There was also a slight general bias for model input temperature to be cooler than observed, counter to the direction expected to lead to under-modeling of SWE. There was also under-modeling of SWE for a subset of sites where precipitation inputs were good. Furthermore, the NWM generally tends to melt snow early. There was considerable variability between modeled and observed SCAF as well as the binary comparison of snow cover presence that hampered useful interpretation of SCAF comparisons. This is in part due to the shortcomings associated with both model SCAF parameterization and MODIS observations, particularly in vegetated regions. However, when SCAF was aggregated across all sites and years, modeled SCAF tended to be more than observed using MODIS. These differences are regional with generally better SWE and SCAF results in the Central Basin and Range and differences tending to become larger the further away regions are from this region. These findings identify areas where predictions from the NWM involving snow may be better or worse, and suggest opportunities for research directed towards model improvements.
\end{abstract}

This study compares the U.S. National Water Model (NWM) reanalysis snow outputs to observed snow water equivalent (SWE) and snow-covered area fraction (SCAF) at SNOTEL sites across the Western U.S. This was done to evaluate and identify opportunities for improving the modeling of snow in the NWM. SWE was obtained from SNOTEL sites, while SCAF was obtained from MODIS observations at a nominal 500 $\mathrm{m}$ grid scale. Retrospective NWM results were at a $1000 \mathrm{~m}$ grid scale. We compared results for SNOTEL sites to gridded NWM and MODIS outputs for the grid cells encompassing each SNOTEL site. Differences between modeled and observed SWE were attributed to both model errors, as well as errors in inputs, notably precipitation and temperature. The NWM generally under-predicted SWE, partly due to precipitation input differences. There was also a slight general bias for model input temperature to be cooler than observed, counter to the direction expected to lead to under-modeling of SWE. There was also under-modeling of SWE for a subset of sites where precipitation inputs were good. Furthermore, the NWM generally tends to melt snow early. There was considerable variability between modeled and observed SCAF that hampered useful interpretation of these comparisons. This is in part due to the model grid SCAF essentially being binary (snow or no snow) while observations from MODIS are much more fractional. However, when SCAF was aggregated across all sites and years, modeled SCAF tended to be more than observed using MODIS. These differences are regional with generally better SWE and SCAF results in the Central Basin and Range and differences tending to become larger the further away regions are from this region. These findings identify 
areas where predictions from the NWM involving snow may be better or worse, and suggest opportunities for research directed towards model improvements.

\section{Hosted file}

Garousi-Nejad_Tarboton_MainText_Authorea.pdf available at https://authorea.com/users/ 279297/articles/515103-a-comparison-of-national-water-model-retrospective-analysis-snowoutputs-at-snotel-sites-across-the-western-u-s 